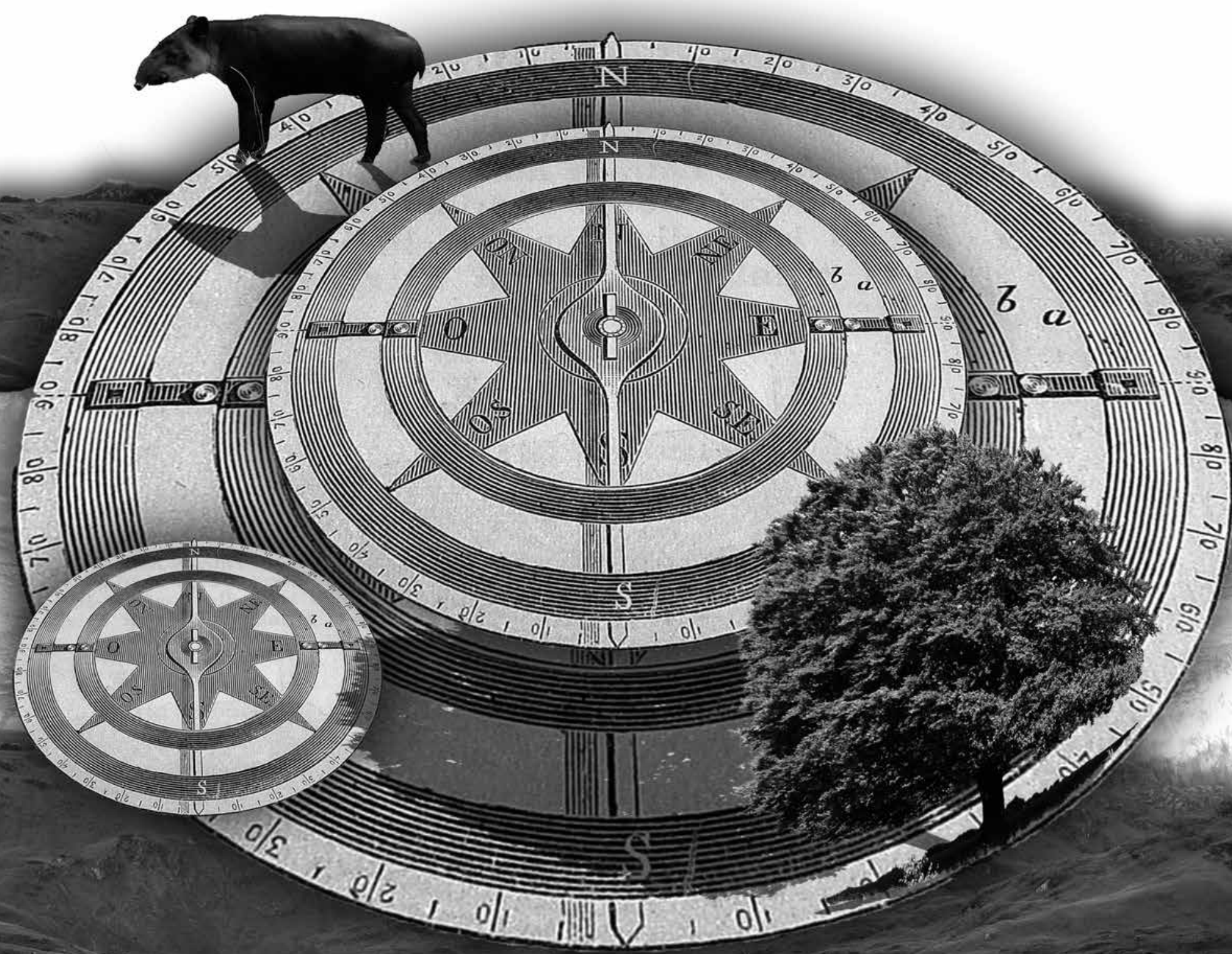

\title{
Hacer turismo ecológico de reconocimiento a la zona rural
}

Make recognition eco tourism the rural area

Fazendo eco turismo reconhecimento a rural Javier Alfonso Delgadillo Molano Egnan Yesid Álvarez Cruz Claudia Patricia Gallo Castro Henry Wilson León Calderón 


\section{Javier Alfonso Delgadillo Molano' \\ Egnan Yesid Álvarez $\mathrm{Cruz}^{2}$ Claudia Patricia Gallo Castro ${ }^{3}$ Henry Wilson León Calderón ${ }^{4}$}

1. Licenciado en Danzas y Teatro, Especialista en Arte y Folclor y Especialista en Gerencia de Proyectos; Máster en Gestión Cultural, candidato a Doctor en Cultura y Educación en América Latina (UARCIS, Santiago-Chile, 2014).

2. Licenciado en Danzas y Teatro, Especialista en docencia del Juego Coreográfico. Magister en Educación (2010).

3. Licenciada en Danza y Teatro, Magister en Discapacidad e Inclusión social (2012). Correo electrónico: paticogallo@gmail.com

4. Licenciado en Danzas y Teatro, Magister en Investigación Social Interdisciplinaria; Especialista en Multimedia Educativa y en Educación Artística del Centro de Altos Estudios Universitarios de la OEI (2013).

Fecha de recepción: 13 de marzo de 2015 / Fecha de aprobación: 30 de octubre de 2015

\section{Resumen}

La presente reflexión tiene que ver con el quehacer del maestro rural en la construcción de muestras escénicas y artísticas que recrean y apropian prácticas surgidas en territorios rurales, las cuales, sumadas a iniciativas de organización comunitaria, industria cultural o de economía creativa, muestran la ruta para la creación de un proyecto de ecoturismo rural. La forma de potenciar y reunir los saberes construidos por la relación entre sujeto y territorio se evidencia en la estrategia propuesta.

Palabras clave: Ecoturismo rural, territorio, saber tradicional, industria cultural.

\section{Summary}

This reflection has to do with the work of the rural teacher in the creation of scenic and artistic performances that recreate and embrace practices emerged in rural territories. In conjunction with community organization initiatives, cultural industry and creative economy initiatives, these practices show the way of creating a project of rural ecotourism. The way of gathering and developing the knowledge acquired in the relationship between subject and territory becomes evident in the strategy suggested by the authors.

Key words: Rural ecotourism, territory, traditional knowledge and cultural industry.

\section{Resumo}

Esta reflexão diz respeito ao trabalho do professor rural na construção de performances cênicas e artísticas que recriam e se apropriam de práticas surgidas nos territórios rurais e que juntamente com iniciativas de organização comunitária, de indústria cultural ou de economia criativa indicam o caminho para a criação de um projeto de ecoturismo rural. A maneira de acompanhar e reunir os saberes construídos pela relação sujeito e território é evidente na estratégia proposta.

Palavras chave: Ecoturismo rural, território, conhecimento tradicional, indústria cultural. 
El siguiente ensayo es producto de la ponencia seleccionada para alimentar el nodo temático 1 del "III Encuentro Iberoamericano en Políticas, Gestión e Industrias Culturales. "Encuentro y diálogos entre culturas" desarrollado en Santiago de Chile en el mes de mayo; se inscribe en la convocatoria del IDEP sobre la temática Escuela, memoria y territorio y contiene la reflexión pedagógica de la articulación escuela-territorio para las Zonas de Interés Turístico (ZIT), específicamente de Ciudad Bolívar, Bogotá, Colombia.

La ponencia relata la estrategia que divisamos como maestros para "Hacer turismo ecológico de reconocimiento a la zona rural", uniendo el saber tradicional, recreando la expresión dancística y musical que se produce en la escuela rural, y sistematizando las organizaciones comunitarias. La estrategia se propone dar la ruta de acompañamiento a organizaciones culturales rurales que inician un proyecto eco turístico; la proyección que jóvenes, niños y adultos hagan en el contexto, permitirá que se reconozcan en su experiencia, trayectoria y saber elaborado en y con el territorio.

Atendiendo a los conceptos emergentes de las relaciones e interrelaciones del ser, la cultura, el contexto, y reconociendo la vida expresiva constituida por el diálogo con el territorio rural, se dan cita diferentes agentes capaces de sorprenderse ante el hecho estético y la producción cultural en el territorio, en una armónica unión, juntos, personas y contextos, se deben descubrir para construir un servicio turístico desde su comprensión y re-creación del mundo. Dándole a las producciones cotidianas un componente escénico y cultural para propender por el eco turismo.

Los nuevos acontecimientos en el devenir político del país, la responsabilidad en la restauración del tejido social, la equidad y el papel fundamental de la educación en el tránsito para alcanzar la paz, llevaron a que los maestros-investigadores socializaran, en forma de ensayo, la ponencia que puede mostrar algunos caminos.

"No hay memoria sin territorio", es una frase dentro de la convocatoria del IDEP y es, para los escritores-maestros-reflexivos, un enunciado que valida la propuesta que aquí se pone en consideración. ¿Cómo la escuela apropia el contexto y lo transforma? Es la pregunta que abre el panorama para generar ámbitos para el debate, estos son: la creación y la puesta en marcha de procesos de reflexión, transformación de las relaciones entre Estado y sociedad civil, y nuevos modelos de políticas públicas de apoyo a la gestión de las industrias culturales dispuestas en el territorio rural.
Vale la pena decir, antes de iniciar la muestra de la ruta, que los maestros que aquí escriben han apreciado el proceso que se desarrolla en la institución educativa José Celestino Mutis, han sido jurados en varios de sus eventos, como la semana cultural, entre otros, donde el territorio y la escuela parecen uno solo, su participación decidida y regular en las primeras versiones del Festival de Danza Delia Zapata Olivella, ha permitido apreciar su compromiso con la tradición y la reivindicación del campesino y su importante labor en el territorio.

La constante inquietud que suscita en los maestros el hecho escénico, y su trascendencia en el desarrollo del conocimiento, permite también vincular el saber producido en la escuela con la comprensión y la importancia del contexto; las prácticas que desarrollan están en constante reflexión e interacción con múltiples saberes, he aquí la evidencia que se llevará a Chile.

\section{Innovación y oportunidad}

El carácter de innovación de la estrategia asume y abarca dos direcciones complementarias, que se sustentan ampliamente: en la normatividad existente para la creación de proyectos de turismo rural y en el desarrollo de aprendizajes significativos desde la escuela.

Teniendo en cuenta que el territorio, y algunas de las trayectorias sugeridas como posibles recorridos en las rutas eco-turísticas, colinda con terrenos de empresas dedicadas a la producción de bienes, que han impactado y transformado el medio, se plantea, dentro de la estrategia, que dichas compañías sea copartícipes y asuman acciones de restitución y de responsabilidad social, apoyando programas de educación ambiental, de responsabilidad ambiental y/o de ejercicio del derecho cultural, así como el apoyo a las investigaciones que realicen las organizaciones de la comunidad en torno al tema eco-turístico. Las instituciones educativas se configuran como el semillero de las industrias culturales y estarán en permanente diálogo con la producción de escenas tradicionales y recontextualizadas en música, teatro y danza.

La propuesta se sustenta teórica e históricamente desde una investigación previa sobre el tema eco-turístico, teniendo en cuenta diferentes referentes conceptuales y legales, entre otros, el Código Mundial para el Turismo (2001), que enuncia la contribución de esta actividad al entendimiento y al respeto mutuos entre hombres y sociedades (Artículo 1); su importancia como instrumento de desarrollo personal y colectivo (Artículo 2); su 
papel en tanto factor de desarrollo sostenible (Artículo 3); y su lugar en el aprovechamiento y enriquecimiento del patrimonio cultural de la humanidad (Artículo 4).

También se emplea el documento de Fernanda Maciel (2009) sobre la formación de ciudadanos capaces de responder a la necesidad de lograr un equilibrio entre el ser humano y el ambiente que lo rodea. A este se suma la consulta del Plan Maestro de Turismo para Bogotá, D.C., Decreto 664 de 2011, en el cual se establecen las pautas para el desarrollo turístico de la ciudad, se muestran claras ideas para gestionar, desde la perspectiva turística, el suelo urbano y rural de Bogotá D.C., y para hacer productivo su uso bajo principios de equilibrio territorial, socio económico y ambiental (Articulo 1).

En el mismo Plan Maestro se autoriza a promover la gestión y financiación público-privada mediante la conformación de agremiaciones cívico-privadas (Articulo 5), y se considera la viabilidad de estos proyectos en los espacios rurales dentro del territorio del Distrito Capital; en el suelo rural se promoverá el desarrollo de acciones de turismo rural, agroturismo y ecoturismo, como principales tipologías de la actividad, con espacios para su desarrollo (Articulo 11), incluyen los lugares de bella apreciación como Zonas de Interés Turístico (ZIT).

Por otra parte, fue necesario consultar la Política para el desarrollo del turismo comunitario en Colombia, en la que se tiene en cuenta que uno de sus objetivos para esta actividad es impulsar la planificación de emprendimientos de turismo comunitario acordes con las estrategias de gestión locales y los procesos de ordenamiento territorial (Numeral 9.2.1, Objetivos específicos); también se busca propiciar la participación de las comunidades con vocación hacia el turismo en la elaboración de los planes de ordenamiento territorial, para definir las zonas de desarrollo turístico prioritarias, en las cuales las distintas organizaciones deseen desarrollar sus proyectos turísticos (Literal B, Numeral, 9.3. Planificación del Turismo Comunitario).

En el mismo sentido, la política busca facilitar la creación de estrategias y orientaciones para el turismo comunitario en las regiones, de acuerdo con el diagnóstico de proyectos identificados en cada región (Literal C, Numeral 9.3), y adecuar las condiciones para diseñar planes de capacitación dirigidos a la creación de productos turísticos comunitarios competitivos, acordes con las realidades y particularidades socioeconómicas, culturales y ambientales, que estimulen la autogestión (Literal D, Numeral 9.3), mientras, al tiempo se propician espacios de diálogo para intercambiar experiencias nacionales e internacionales de emprendimientos de turismo comunitario, que permitan la capacitación continua de las comunidades bajo el análisis de lecciones aprendidas (Literal E, Numeral 9.3).

Por otra parte, fue necesario revisar el Plan Decenal de Educación, pues tiene en saldo rojo temas que toman cuerpo en la presente estrategia, y que son elementos fundantes, a pesar de que, aunque son prioritarios en el Plan, en su mayoría cuentan con un menor desarrollo dentro de la medición del Ministerio de Educación Nacional ante los adelantos de las diferentes Secretarías de Educación. Dichos criterios de articulación son:

- El impulso a la educación para la paz, los Derechos Humanos, la convivencia y la ciudadanía (Numeral 7).

- El impulso a la educación ambiental en las IE (Numeral 8).

- El fomento de la investigación y la innovación en las instituciones educativas (Numeral 9).

- La articulación de la formación disciplinar con las artes, la cultura y el deporte (Numeral 10).

- El fomento de la articulación de la educación media con la superior y con la educación para el trabajo y el desarrollo humano, haciendo promoción de estrategias para fomentar el emprendimiento en los establecimientos educativos (Numeral 12).

- El impulso a la efectiva vinculación de la familia a la institución educativa desde una perspectiva de corresponsabilidad (Numeral 22).

- El impulso a mecanismos de participación y corresponsabilidad con la educación de los sectores productivo y solidario (Numeral 23).

La oportunidad de las acciones está directamente ligada al Plan de Acción y al marco legislativo vigente, para la ciudad y el país, en torno al tema ecoturístico; todo ello sustenta el proyecto y el carácter investigativo que debe tener. Al mismo tiempo, es necesario considerar la manera en que se estructura el Plan de Acción con las organizaciones del territorio, para que se hagan claramente evidentes los componentes de la innovación, en: la misión, la visión, los objetivos, las estrategias, las acciones y la propuesta investigativa en la que se incluya el componente social, el compromiso educativo, cultural, ambiental y de patrimonio como industria cultural.

En un primer momento se debe caracterizar a los grupos, identificando su experiencia ecoturística en la zona, mediante el contacto y diálogo directo, las articulaciones con el sector educativo y con los maestros de artes escénicas; luego se deben validar las rutas y saberes ecoturísticos constituidos por cada 


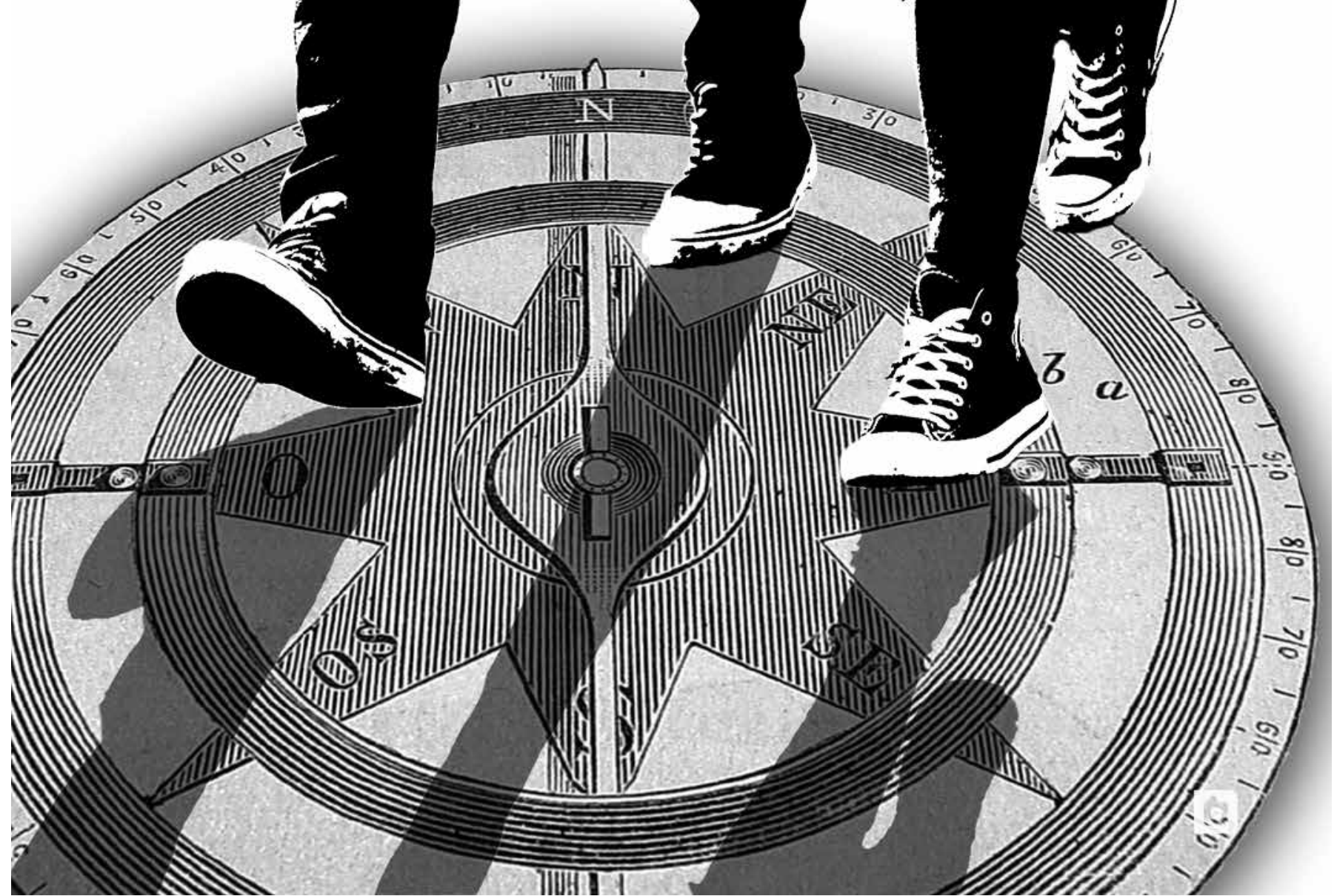

organización rural, para definir las líneas de acción comunes a las organizaciones encontradas, identificando oportunidades y fortalezas para la elaboración del Plan de Acción que permita promover y desarrollar actividades eco turísticas, estableciendo el objeto social (educación, responsabilidad social, responsabilidad ambiental, derecho cultural).

En un segundo momento se implementan pruebas piloto con entidades educativas, culturales y/o económicas, identificadas como potenciales usuarios de las rutas ecoturísticas diseñadas, de tal manera que sea posible levantar el estudio de impacto y satisfacción del servicio ecoturístico.

El resultado permite establecer las estrategias de mercadeo con la participación directa de las comunidades, para la promoción y proyección de servicios de turismo cultural en los sectores rurales periféricos de la ciudad capital, desde el enfoque de responsabilidad social que permita incrementar el grado de compromiso y permanencia de la identidad social y cultural, que se sumará a los lenguajes escénicos de tradiciones, configurados en las clases de artes escénicas en las IEDs rurales.

Los resultados esperados, y de obligatoria producción para la viabilidad de la estrategia, son:

1. Documento de caracterización de los grupos y organizaciones comunitarias, entre ellas, las instituciones educativas.

2. Documento que contenga la definición del plan estratégico de las organizaciones, la vinculación de empresas copartícipes del territorio, y la línea de investigación surgida en el proceso.

3. Formatos de evaluación del impacto de satisfacción del servicio ecoturístico.

4. Brochure o dossier del servicio ecoturístico ofertado por parte de las organizaciones.

Indicadores previstos para medir la eficacia de la estrategia:

1. Grupos caracterizados / total de grupos encontrados.

$2.100 \%$ de las experiencias sistematizadas.

3. Recorridos seleccionados / Recorridos propuestos.

4. Maestros de artes escénicas vinculados / maestros de artes.

5. Instituciones educativas vinculadas / Instituciones educativas del sector.

La alianza entre el Centro de Investigación de las Artes escénicas, CIAES, y La Asociación de Licenciados de Danzas y Teatro, ASOLDYT, nutre la visión de los maestros que arman la estrategia y han obtenido, como insumo, el resultado de varias versiones del Festival de Danza y Teatro Escolar Delia Zapata Olivella (doce versiones hasta 2014) y del Encuentro de Teatro Escolar “entrEscena” (decima versión en 2014) en la ciudad de Bogotá D.C. Los eventos han mostrado grupos 


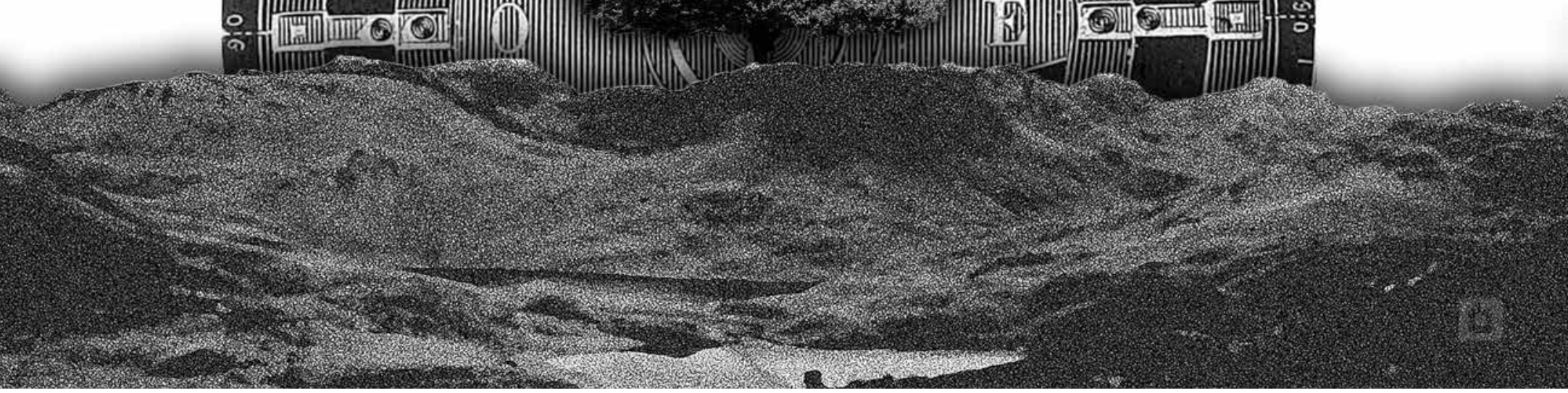

de estudiantes de los Colegios rurales y urbanos, entre otros, recreando y retornando a prácticas patrimoniales, que bien pueden unirse a dinámicas comunitarias de creación de ecoturismo rural con escenificaciones propias del territorio.

Otro aspecto a resaltar es la pertinencia de la estrategia dentro del Plan Maestro de Turismo de Bogotá D.C. (2011), donde se plantea, dentro del marco lógico, el programa de valoración del componente rural de la ciudad, para la promoción y gestión de su uso turístico sostenible, concretándose en actividades que buscan mejorar los atractivos y recursos turísticos de este tipo de suelo, acción que involucra a la zona rural de Ciudad Bolívar y a otras zonas periféricas.

El conocimiento de las organizaciones comunitarias presentes en las veredas, y con experiencia de trabajo en el territorio, de los senderos ecológicos, del territorio rural y de las acciones de restitución y promoción que buscan el mejorar y conservar el medio ambiente, es una ventaja que ayudará a la hora de convocar y ensamblar los saberes; también es útil ubicar las organizaciones y grupos artísticos y de investigación sobre tradiciones rurales, para la puesta en escena, principalmente en teatro y danza, en escuelas de formación artística donde circule el saber recuperado, preservado y adquirido con la comunidad infantil y juvenil del sector.

Este tipo de organizaciones deberán recibir un reconocimiento y un apoyo externo, para lograr impulsar sus proyectos en el territorio; si no están organizadas hay que verificar el tipo de estructura existente $y$, con el apoyo de la comunidad, acompañar y orientar estas formas básicas de apropiación de saberes para el eco-turismo sostenible. Las entidades pueden estar interesadas en constituir un convenio de apoyo mutuo para diseñar y llevar a cabo otros proyectos ecoturísticos, teniendo en cuenta que el territorio considerado como Zona de Interés Turístico ZIT (Decreto 664 de 2011) ocupa más de un 50\% de la capital y tiene un gran potencial de desarrollo auto gestionable.

Los beneficiarios directos son las personas que conforman las organizaciones del proyecto, los indirectos serían otras organizaciones del territorio y los oferentes de bienes y servicios. La población usuaria puede aumentar y se calculará de acuerdo a los resultados de evaluación y validación del pilotaje, considerando la capacidad de atención que se pueda ofrecer según las variables de calidad y satisfacción del servicio por parte de los usuarios.

La socialización es parte vital dentro de la estrategia, tiene tres etapas que abarcan a diferentes personas o entidades del entorno próximo al territorio de influencia, hasta llegar a los potenciales consumidores de las rutas turísticas. Cada etapa tiene un objetivo definido de acuerdo al estado de desarrollo en el que se encuentre el sector.

- Etapa uno: Difusión a las empresas, entidades y personas que se encuentran en los márgenes cercanos de los posibles recorridos. Se busca concientizar sobre las medidas de seguridad y los beneficios directos e indirectos de enriquecer el servicio y fortalecer el proyecto como una construcción colectiva de los habitantes, propietarios y, en general, de los agentes mediadores internos y externos al territorio de influencia.

- Etapa dos: Concientización de los procesos, procedimientos, responsabilidades y condiciones de acción, de los agentes mediadores internos y externos involucrados en el proyecto. El objetivo es considerar las diferentes variables a tener en cuenta en las rutas eco-turísticas diseñadas, para atenuar las dificultades que surjan y garantizar un servicio 
de calidad que satisfaga las expectativas y necesidades de los consumidores, minimizando el riesgo de dificultad.

- Etapa tres: Mercadeo y promoción, es la difusión que se hace a los potenciales consumidores, con quienes se harán prácticas de validación y se aplicarán instrumentos de evaluación para realizar los ajustes correspondientes. El objetivo es hacer un estudio con la población interesada en contribuir a preservar, cuidar y aprender, a través de la experiencia directa en el sector cultural rural periurbano de Bogotá, Colombia.

En todos los casos se debe tener un programa de difusión de los avances con los pobladores y vecinos, ya que se busca mantener el respeto por la manera como esto afecta su realidad, en procura de empoderarlos y consolidar la sostenibilidad del proyecto eco-turístico. Esta estrategia para acompañar y orientar el proyecto ecoturístico rural, asume a la comunidad como actor y beneficiario del proceso del turismo cultural, y promueve los emprendimientos turísticos comunitarios basados en su cultura, para la superación de su situación de pobreza y la difusión de la inclusión y la dignificación de la labor campesina, de su aporte artístico y cultural.

Todo ello promueve la innovación en turismo cultural y ecológico a partir de iniciativas de economía creativa, desde la activación del patrimonio cultural, la producción artística y las industrias culturales y creativas de los campesinos y campesinas habitantes de los sitios inscritos en la Lista del Patrimonio Mundial de la UNESCO, con actividades expresivas y manifestaciones inscritas en la Lista del Patrimonio Inmaterial de la UNESCO.

\section{Referencias}

Alcaldía Mayor de Bogotá. (2011). Plan Maestro de Turismo para Bogotá, D.C. Decreto 664 de 2011. Obtenido desde http://www.alcaldiabogota.gov.co/sisjur/normas/Norma1.jsp?i=45173

Maciel, Fernanda. (2009). Educación ambiental y turismo. Una formación holística, interdisciplinaria y de futuros educadores. Obtenido desde http://www.scielo.org.ar/scielo.php?script=sci_arttext\&pi$\mathrm{d}=$ S1851-17322009000100006

Ministerio de Comercio, Industria y Turismo. (2012). Politica para el desarrollo del turismo comunitario en Colombia. Obtenido desde http:/ / www.mincit.gov.co/minturismo/descargar.php?id=64384
Ministerio de Educación Nacional. (2006). Plan Decenal de Educación. Obtenido desde http://www.plandecenal.edu.co/html/1726/ w3-channel.html

ONU, Organización Mundial de turismo. (2001). Código ético mundial para el turismo. Obtenido desde http://www.conaculta.gob.mx/turismocultural/documentos/pdf/codigo_etico_OMT.pdf 


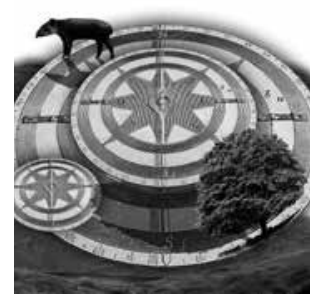

